PROCEEDINGS OF THE

AMERICAN MATHEMATICAL SOCIETY

Volume 126, Number 1, January 1998, Pages 153-157

S 0002-9939(98)04008-8

\title{
NORM ATTAINING FUNCTIONALS ON $C(T)$
}

\author{
P. S. KENDEROV, W. B. MOORS, AND SCOTT SCIFFER \\ (Communicated by Dale Alspach)
}

\begin{abstract}
It is shown that for any infinite compact Hausdorff space $T$, the Bishop-Phelps set in $C(T)^{*}$ is of the first Baire category when $C(T)$ has the supremum norm.
\end{abstract}

For a Banach space $X$ with closed unit ball $B(X)$, the Bishop-Phelps set is the set of all functionals in the dual $X^{*}$ which attain their supremum on $B(X)$; that is, the set $\left\{\mu \in X^{*}\right.$ : there exists $x \in B(X)$ with $\left.\mu(x)=\|\mu\|\right\}$. It is well known that the Bishop-Phelps set is always dense in the dual $X^{*}$. It is more difficult, however, to decide whether the Bishop-Phelps set is residual as this would require a concrete characterisation of those linear functionals in $X^{*}$ which attain their supremum on $B(X)$. In general it is difficult to characterise such functionals, however it is possible when $X=C(T)$, for some compact $T$, with the supremum norm. The aim of this note is to show that in this case the Bishop-Phelps set is a first Baire category subset of $C(T)^{*}$.

A problem of considerable interest in differentiability theory is the following.

If the Bishop-Phelps set is a residual subset of the dual $X^{*}$, is the dual norm necessarily densely Fréchet differentiable?

It is known, for example, that this is the case if the dual $X^{*}$ is weak Asplund, [1, Corollary 1.6(i)], or if $X$ has an equivalent weakly mid-point locally uniformly rotund norm and the weak topology on $X$ is $\sigma$-fragmented by the norm, [2, Theorems 3.3 and 4.4]. It is also known that each dual norm on $X^{*}$ is densely Fréchet differentiable on $X^{*}$ provided that the Bishop-Phelps set of each equivalent norm on $X$ is residual in $X^{*},[6]$. No counterexamples are known, and a consequence of our result is that no space of the form $X=C(T)$ can be a counterexample to the problem above.

Let $T$ be an infinite compact Hausdorff space and $C(T)$ be the space of all continuous real-valued functions on $T$ with the supremum norm $\|x\| \equiv \sup \{|x(t)|$ : $t \in T\}$. We will denote by $B$ the unit ball of $C(T)$; that is, $B \equiv\{x \in C(T):\|x\| \leq$ $1\}$.

For every pair of real-valued functions on $T$ (not necessarily continuous) we write $x \leq y$ if and only if $x(t) \leq y(t)$ for every $t \in T$. We denote by $1_{U}$ the characteristic function of a subset $U$ of $T$; that is,

$$
1_{U}(t)= \begin{cases}1 & \text { if } t \in U \\ 0 & \text { if } t \notin U\end{cases}
$$

Received by the editors December 18, 1995 and, in revised form, June 24, 1996. 1991 Mathematics Subject Classification. Primary 46E15.

(C)1998 American Mathematical Society 
Given $\lambda \in C(T)^{*}$ and an open $U \subset T$ we define

$$
\lambda_{+}^{*}(U) \equiv \sup \left\{\lambda(h): h \in C(T), 0 \leq h \leq 1_{U}\right\} .
$$

Clearly $\lambda_{+}^{*}(U) \geq 0$. Similarly we define

$$
\begin{aligned}
\lambda_{-}^{*}(U) & \equiv \sup \left\{\lambda(-h): h \in C(T), 0 \leq h \leq 1_{U}\right\} \\
& =\sup \left\{(-\lambda)(h): h \in C(T), 0 \leq h \leq 1_{U}\right\} \\
& =(-\lambda)_{+}^{*}(U) .
\end{aligned}
$$

For our first result we will need to consider the following closed sets,

$$
\begin{aligned}
& S_{+}^{\lambda} \equiv\left\{t \in T: \lambda_{+}^{*}(U)>0 \text { for every open neighbourhood } U \text { of } t\right\}, \\
& S_{-}^{\lambda} \equiv\left\{t \in T: \lambda_{-}^{*}(U)>0 \text { for every open neighbourhood } U \text { of } t\right\} .
\end{aligned}
$$

Theorem 1. A linear functional $\lambda \in C(T)^{*}$ attains its supremum on $B$ at $x_{0}$ if and only if

$$
x_{0}(t)= \begin{cases}1 & \text { for every } t \in S_{+}^{\lambda}, \\ -1 & \text { for every } t \in S_{-}^{\lambda} .\end{cases}
$$

That is, $\lambda$ is in the Bishop-Phelps set if and only if $S_{+}^{\lambda} \cap S_{-}^{\lambda}=\varnothing$.

Proof. Suppose $\lambda$ attains its supremum over $B$ at $x_{0}$ but there exists a $t_{0} \in S_{+}^{\lambda}$ such that $x_{0}\left(t_{0}\right)<r<1$. Then there is an open neighbourhood $U_{0}$ of $t_{0}$ such that $x_{0}(t)<r<1$ for every $t \in U_{0}$, by the continuity of $x_{0}$. Since $t_{0} \in S_{+}^{\lambda}$ we have $\lambda_{+}^{*}\left(U_{0}\right)>0$. Then there exists some $h \in C(T), 0 \leq h \leq 1_{U_{0}}$, with $\lambda(h)>0$. Take some $0<\epsilon<1-r$ and consider the function $x_{0}+\epsilon h$. Then

$$
\left(x_{0}+\epsilon h\right)(t)= \begin{cases}x_{0}(t)+\epsilon h(t)<r+\epsilon<1 & \text { for } t \in U_{0}, \\ x_{0}(t) & \text { for } t \notin U_{0} .\end{cases}
$$

Remembering $h \geq 0$ we conclude that $x_{0}+\epsilon h \in B$. On the other hand

$$
\lambda\left(x_{0}+\epsilon h\right)=\lambda\left(x_{0}\right)+\epsilon \lambda(h)>\lambda\left(x_{0}\right)
$$

contradicting the assumption that $\lambda$ attained its supremum on $B$ at $x_{0}$. Hence $x_{0}\left(t_{0}\right)=1$ for every $t_{0} \in S_{+}^{\lambda}$.

Similarly, or by noting that $\lambda_{-}^{*}=(-\lambda)_{+}^{*}$, we conclude that $x_{0}\left(t_{0}\right)=-1$ for every $t_{0} \in S_{-}^{\lambda}$.

Conversely, suppose

$$
x_{0}(t)= \begin{cases}1 & \text { for every } t \in S_{+}^{\lambda}, \\ -1 & \text { for every } t \in S_{-}^{\lambda}\end{cases}
$$

and $y_{0} \in B$. Set

$$
\left(y_{0}-x_{0}\right)^{+}(t)=\max \left\{\left(y_{0}-x_{0}\right)(t), 0\right\}
$$

and

$$
\left(y_{0}-x_{0}\right)^{-}(t)=\min \left\{\left(y_{0}-x_{0}\right)(t), 0\right\}
$$


so $\left(y_{0}-x_{0}\right)=\left(y_{0}-x_{0}\right)^{+}+\left(y_{0}-x_{0}\right)^{-}$. Consider $T_{\epsilon}=\left\{t \in T:\left(y_{0}-x_{0}\right)^{+}(t) \geq\right.$ $\epsilon\}$, a compact subset of $T$ disjoint from $S_{+}^{\lambda}$. For each $t \in T_{\epsilon}$ there is an open neighbourhood $U$ of $t$ for which $\lambda_{+}^{*}(U)=0$. These form an open cover of $T_{\epsilon}$, and so there is a finite subcover $U_{i}$ of $T_{\epsilon}$ for $i=1,2, \ldots, n$, which together with $U_{0} \equiv\left(T_{\epsilon}\right)^{c}$ form an open cover of $T$. Let $p_{i}$ be a partition of unity subordinate to this cover, $[5$, p. 171]. Thus

$$
\begin{aligned}
\lambda\left(\left(y_{0}-x_{0}\right)^{+}\right) & =\lambda\left(\sum_{i=0}^{n} p_{i}\left(y_{0}-x_{0}\right)^{+}\right) \\
& \leq \lambda\left(p_{0}\left(y_{0}-x_{0}\right)^{+}\right) \\
& \leq\|\lambda\|\left\|p_{0}\left(\left(y_{0}-x_{0}\right)^{+}\right)\right\| \\
& \leq\|\lambda\| \epsilon .
\end{aligned}
$$

Similarly $\lambda\left(\left(y_{0}-x_{0}\right)^{-}\right) \leq\|\lambda\| \epsilon$, and so we conclude that $\lambda\left(y_{0}-x_{0}\right) \leq 2\|\lambda\| \epsilon$. Since this is true for any $\epsilon>0$ we have $\lambda\left(y_{0}\right) \leq \lambda\left(x_{0}\right)$ for all $y_{0} \in B$, so $\lambda$ attains its supremum over $B$ at $x_{0}$.

We need one more definition to formulate our next proposition. Given $\lambda \in C(T)^{*}$ and $t \in T$, define

$$
\begin{aligned}
& r_{+}^{\lambda}(t)=\inf \left\{\lambda_{+}^{*}(U): t \in U, U \text { open }\right\} \text { and } \\
& r_{-}^{\lambda}(t)=\inf \left\{\lambda_{-}^{*}(U): t \in U, U \text { open }\right\} .
\end{aligned}
$$

Theorem 2. For every $\lambda \in C(T)^{*}$ the sets $\left\{t \in T: r_{+}^{\lambda}(t)>0\right\}$ and $\{t \in T$ : $\left.r_{-}^{\lambda}(t)>0\right\}$ are countable. Moreover, $\sum_{t \in T} r_{+}^{\lambda}(t)<\infty$ and $\sum_{t \in T} r_{-}^{\lambda}(t)<\infty$.

Proof. Let $t_{i} \in T$ for $i=1,2, \ldots, n$, be distinct points in $T$ and let $\epsilon>0$. There exist disjoint open sets $U_{i}$ such that $t_{i} \in U_{i}$. Since $\lambda_{+}^{*}\left(U_{i}\right) \geq r_{+}^{\lambda}\left(t_{i}\right)>r_{+}^{\lambda}\left(t_{i}\right)-\epsilon / 2^{i}$, there exists some $h_{i} \in C(T)$, with $0 \leq h_{i} \leq 1_{U_{i}}$, such that $\lambda\left(h_{i}\right)>r_{+} \lambda\left(t_{i}\right)-\epsilon / 2^{i}$. Then $h \equiv \sum_{i=1}^{n} h_{i}$ is in $B$ since the open sets $U_{i}$ are a disjoint family. Thus

$$
\|\lambda\| \geq \lambda(h)=\sum_{i=1}^{n} \lambda\left(h_{i}\right)>\sum_{i=1}^{n} r_{+}^{\lambda}\left(t_{i}\right)-\epsilon \sum_{i=1}^{n} \frac{1}{2^{i}} .
$$

Consequently $\|\lambda\|+\epsilon>\sum_{i=1}^{n} r_{+}^{\lambda}\left(t_{i}\right)$, which shows that $\sum_{t \in T} r_{+}^{\lambda}(t)<\infty$, and so the set $\left\{t \in T: r_{\lambda}(t)>0\right\}$ must be countable. That $\sum_{t \in T} r_{-}^{\lambda}(t)<\infty$, and the set $\left\{t \in T: r_{-}^{\lambda}(t)>0\right\}$ is countable, follow similarly.

We note that the points $t \in T$ where $r_{+}^{\lambda}(t)>0\left(r_{-}^{\lambda}(t)>0\right)$ are points with positive (negative) $\lambda$-measure. They are related to the " $l_{1}$-part" of the functional $\lambda$, an $l_{1}$-weighted sum of point evaluations.

Lemma 3. Given $\lambda \in C(T)^{*}$ and $t \in T$ where $r_{+}^{\lambda}(t)>0\left(r_{-}^{\lambda}(t)>0\right)$ then there exists an open neighbourhood $A$ of $\lambda$ such that $r_{+}^{\mu}(t)>0\left(r_{-}^{\mu}(t)>0\right)$ for each $\mu \in A$.

Proof. We consider the case $r_{+}^{\lambda}(t)>0$. Choose $\epsilon>0$ such that $r_{+}^{\lambda}(t)>3 \epsilon$, and consider any $\mu \in A \equiv\left\{\mu \in C(T)^{*}:\|\lambda-\mu\|<\epsilon\right\}$. Let $U$ be any open neighbourhood $t$, then $\lambda_{+}^{*}(U)>3 \epsilon$. Then there exists an $h \in B, 0 \leq h \leq 1_{U}$, such that $\lambda(h)>2 \epsilon$. Since $\|\lambda-\mu\|<\epsilon$ we conclude that $\mu(h)>\epsilon$. Hence $r_{+}^{\mu}(t) \geq \epsilon>0$.

The case $r_{-}^{\lambda}(t)>0$ follows similarly. 
All is in readiness for our main theorem.

Theorem 4. For any infinite compact Hausdorff space T, the Bishop-Phelps set is of the first Baire category in $C(T)^{*}$.

Proof. The proof splits into two cases, $T$ is scattered or not.

Case 1. T scattered. In this case $T$ contains a convergent sequence $\left\{t_{i}\right\}$ of distinct points with limit point $t_{\infty} \in T$, [3, Lemma 5.3]. Consider the sets

$$
\begin{aligned}
& G_{i} \equiv\left\{\mu \in C(T)^{*}: \text { for some } j>i, r_{+}^{\mu}\left(t_{j}\right)>0\right\} \\
& H_{i} \equiv\left\{\mu \in C(T)^{*}: \text { for some } j>i, r_{-}^{\mu}\left(t_{j}\right)>0\right\}
\end{aligned}
$$

Now these are open by Lemma 3. They are also dense since for any $\lambda \in C(T)^{*}$ and any $\epsilon>0$ there exists a $j>i$ such that $r_{-}^{\lambda}\left(t_{j}\right)<\epsilon\left(r_{+}^{\lambda}\left(t_{j}\right)<\epsilon\right)$ and so for $\mu(x) \equiv \lambda(x)+\epsilon x\left(t_{j}\right)\left(\mu(x) \equiv \lambda(x)-\epsilon x\left(t_{j}\right)\right)$ we have $r_{+}^{\mu}\left(t_{j}\right)>0\left(r_{-}^{\mu}\left(t_{j}\right)>0\right)$, and so $\mu \in G_{i}\left(\mu \in H_{i}\right)$. But then for any $\lambda \in \bigcap_{i=1}^{\infty}\left(G_{i} \cap H_{i}\right)$, we have $t_{\infty} \in S_{+}^{\lambda} \cap S_{-}^{\lambda}$, and by Theorem $1, \lambda$ does not attain its supremum over $B$. That is, the Bishop-Phelps set lies in the complement of a dense $G_{\delta}$ set, and so is of the first Baire category in $C(T)^{*}$.

Case 2. $T$ not scattered. In this case there exists a continuous function $p$ mapping $T$ onto $[0,1][4, \S 8.5 .4,(\mathrm{i}) \Rightarrow(\mathrm{ii})]$. By Zorn's Lemma there exists a minimal compact subset $T^{\prime}$ of $T$ such that $p\left(T^{\prime}\right)=[0,1]$. Let $\left\{V_{i}\right\}$ be a countable open base for the interval $[0,1]$ and define the sets

$$
\begin{aligned}
& G_{i} \equiv\left\{\mu \in C(T)^{*}: \text { for some } t \in p^{-1}\left(V_{i}\right) \cap T^{\prime}, r_{+}^{\mu}(t)>0\right\}, \\
& H_{i} \equiv\left\{\mu \in C(T)^{*}: \text { for some } t \in p^{-1}\left(V_{i}\right) \cap T^{\prime}, r_{-}^{\mu}(t)>0\right\} .
\end{aligned}
$$

Again by Lemma 3 these sets are open. Since each set $p^{-1}\left(V_{i}\right) \cap T^{\prime}$ is uncountable, for any $\lambda \in C(T)^{*}$ there exists a $t \in p^{-1}\left(V_{i}\right) \cap T^{\prime}$ such that $r_{+}^{\lambda}(t)=0\left(r_{-}^{\lambda}(t)=0\right)$. So for any $\epsilon>0$, and $\mu(x) \equiv \lambda(x)+\epsilon x(t)(\mu(x) \equiv \lambda(x)-\epsilon x(t))$, we have $r_{+}^{\mu}(t)>0$ $\left(r_{-}^{\mu}(t)>0\right)$, and so $\mu \in G_{i}\left(\mu \in H_{i}\right)$. That is, $G_{i}$ and $H_{i}$ are dense.

Consider any $\lambda \in \bigcap_{i=1}^{\infty}\left(G_{i} \cap H_{i}\right)$. Let $t \in T^{\prime}$ and $N$ be an open neighbourhood of $t$. Since $T^{\prime}$ is minimal, $p\left(T^{\prime} \backslash N\right) \neq[0,1]$, and so we may choose $V_{n} \subset[0,1] \backslash p\left(T^{\prime} \backslash N\right)$. Thus $p^{-1}\left(V_{n}\right) \cap T^{\prime} \subset N \cap T^{\prime} \subset N$. As $\lambda \in G_{n}\left(H_{n}\right)$ there exists an element $t_{n} \in p^{-1}\left(V_{n}\right) \cap T^{\prime} \subset N$ such that $r_{+}^{\lambda}\left(t_{n}\right)>0\left(r_{-}^{\lambda}\left(t_{n}\right)>0\right)$. In particular, $N \cap S_{+}^{\lambda} \neq \varnothing$ and $N \cap S_{-}^{\lambda} \neq \varnothing$. Now this holds for arbitrary open sets $N$, and the sets $S_{+}^{\lambda}$ and $S_{-}^{\lambda}$ are both closed. We conclude that $t \in S_{+}^{\lambda}$ and $t \in S_{-}^{\lambda}$. That is, $T^{\prime} \subset S_{+}^{\lambda} \cap S_{-}^{\lambda}$, and so by Theorem $1, \lambda$ does not attain its supremum over $B$. That is, the BishopPhelps set lies in the complement of a dense $G_{\delta}$ set, and so is of the first Baire category in $C(T)^{*}$.

\section{REFERENCES}

[1] J. R. Giles, P. S. Kenderov, W. B. Moors, and Scott Sciffer, Generic differentiability of convex functions on the dual of a Banach space, Pacific J. Math. 172 (1996), 413-431. CMP 96:11

[2] J. R. Giles and W. B. Moors, Generic continuity of minimal set-valued mappings, preprint.

[3] I. Namioka, Radon-Nikodým compact space and fragmentability, Mathematika 34 (1987), 258-281. MR 89i:46021 
[4] Z. Semadeni, Banach spaces of continuous functions, Vol. 1, PWN, Warszawa, 1971. MR 45:5730

[5] John L. Kelley, General Topology, D. Van Nostrand, 1955. MR 16:1136c

[6] W. B. Moors, The relationship between Goldstine's theorem and the convex point of continuity property, J. Math. Anal. and Appl. 188 (1994), 819-832. MR 95k:46024

The Institute of Mathematics, Bulgarian Academy of Sciences, Box 373, BG-1090, Sofia, Bulgaria

Department of Mathematics, The University of Auckland, Auckland, New Zealand

Department of Mathematics, The University of Newcastle, New South Wales 2308, Australia 